\title{
ORIGINAL ARTICLE Intramedullary pressure changes in rats after spinal cord injury
}

\author{
X Dong ${ }^{1}$, D Yang ${ }^{1}, \mathrm{~J} \mathrm{Li}, \mathrm{C}$ Liu, M Yang, L Du, R Gu, A Hu and H Zhang
}

Objectives: The objectives of this study were to explore the change of intramedullary pressure over time in rats after different degrees of spinal cord contusion injury and to verify the hypothesis that the more serious the injury, the higher the intramedullary pressure. Methods: The control group rats underwent laminectomy only, whereas the rats in the three experimental groups were subjected to mild, moderate or severe 10th thoracic cord (T10) contusion injury after laminectomy. In addition, an intramedullary pressure of T10 was measured by a Millar Mikro-Tip pressure catheter (Millar Incorporated Company, Houston, TX, USA) immediately in the control group or at different time points after injury in the experimental groups.

Results: The average intramedullary pressure of the rats in the control group was $6.88 \pm 1.67 \mathrm{~mm} \mathrm{Hg}$, whereas that of the rats in any injury group was significantly higher $(P=0.000)$. There was statistical difference among the different time points in the mild or moderate injury group $(P=0.007 / 0.017)$, but no in the severe $(P=0.374)$. The curves of intramedullary pressure over time in the mild and moderate injury group were bimodal, peaking at 1 and $48 \mathrm{~h}$ after the injury. The intramedullary pressure after injury was positively correlated with the injury degree $(r=0.438, P=0.000)$.

Conclusions: The intramedullary pressure of the rats increased after traumatic spinal cord injury. If the injury was not serious, the intramedullary pressure fluctuated with time and peaked at 1 and $48 \mathrm{~h}$ after injury. If the injury was serious, the intramedullary pressure remained high. The more serious the injury, the higher the intramedullary pressure.

Spinal Cord (2016) 54, 947-950; doi:10.1038/sc.2016.35; published online 12 April 2016

\section{INTRODUCTION}

In the acute phase of spinal cord injury, with the constraints of the spinal dura, intramedullary hemorrhage and edema inevitably induce increases in intramedullary pressure. Previous literature has shown that the increased pressure could result in nerve tissue injuries. ${ }^{1-3}$ Therefore, the increase in intramedullary pressure could cause a secondary spinal cord injury. Prompt and effective decreases in intramedullary pressure in the acute phase of spinal cord injury could minimize secondary spinal cord injury. However, the normal intramedullary pressure of the spinal cord, as well as the changes in intramedullary pressure after traumatic spinal cord injury and the correlation between intramedullary pressure and injury degree, are unclear.

This experiment aimed to explore the changes in intramedullary pressure after different degrees of traumatic spinal cord injury in Sprague-Dawley rats and to verify the assumption that the more serious the injury degree, the higher the intramedullary pressure.

\section{METHODS}

Experimental animals and groups

A total of 145 female specific-pathogen-free Sprague-Dawley rats ( 9 weeks old, $250 \mathrm{~g}$ each) were purchased from the Animal Center of Chinese Academy of Military Medical Sciences. The rats were raised in the shielding environment in accordance with the national standard (GB14925-2010).
The animals were randomly divided into the control group (10 rats) and the experimental group (135 rats). The rats in the control group underwent laminectomy only, and an intramedullary pressure of T10 was measured by a Millar Mikro-Tip pressure catheter (SPR-671NR, 1.4 F, Millar Company, USA). The rats in the experimental group were divided into three groups and subjected to mild, moderate or severe spinal cord contusion injury after laminectomy. Each group was further divided into nine subgroups, and the intramedullary pressure was measured at different time points after the injury (10 min, 1 h, 2 h, 4 h, 8 h, 24 h, 48 h, 4 days and 7 days).

\section{Laminotomy}

The rats were anesthetized via an intraperitoneal administration of $2 \%$ pentobarbital sodium at the dose of $3.5 \mathrm{mg} / 100 \mathrm{~g}$ and fixed on the operating table. After skin sterilization, a $2-\mathrm{cm}$ longitudinal incision was made around the midpoint of the T10 spinous process. The adipose layer and fascia were cut. The spinous process and the adjacent muscles were bluntly dissected. The interspinal ligaments between T9 and T12 spinous processes were cut. Bilateral pedicles of both T10 and T11 were cut, and the vertebral laminas were uncovered to expose the spinal cord.

Spinal cord contusion injury model (experimental group only)

The spinal cord contusion injury model was made according to the current international MASCIS Impactor Method. ${ }^{4}$ In this study, the third-generation MASCIS Impactor (W.M. Keck Center for Collaborative Neuroscience, Rutgers State University of New Jersey, Piscataway, NJ, USA) was used. The spine of

Center of Neural Injury and Repair, Beijing Institute for Brain Disorders, Department of Spinal and Neural Function Reconstruction, China Rehabilitation Research Center, Faculty of Rehabilitation Medicine, Capital Medical University, Beijing, China

1These authors contributed equally to this work.

Correspondence: Dr J Li, Center of Neural Injury and Repair, Beijing Institute for Brain Disorders, Department of Spinal and Neural Function Reconstruction, China Rehabilitation Research Center, Faculty of Rehabilitation Medicine, Capital Medical University, No. 10, Jiaomen North Road, Fengtai District, Beijing 100068 , China.

E-mail: crrclijianjun@gmail.com

Received 12 September 2015; revised 24 January 2016; accepted 15 February 2016; published online 12 April 2016 
Table 1 Intramedullary pressure of rats in the experimental groups by damage degree over time

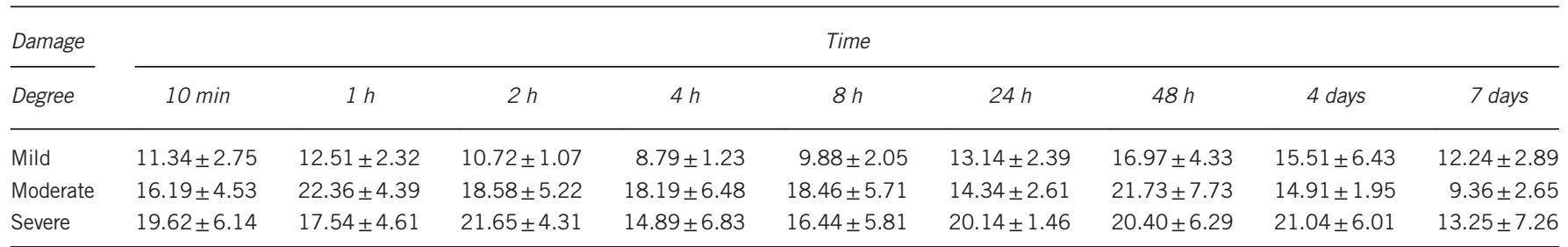

Values are shown as mean \pm s.d. in $\mathrm{mm} \mathrm{Hg}$.

each rat was fixed with spinous process clip to center the T10 spinal cord under the head of the falling rod. The paraspinal muscles of T10 were clipped by an alligator clip. We first dropped the falling rod until it contacted the spinal dura (when the alarm rang), and then calibrated the device. We then lifted the falling rod to a certain height $(12.5 \mathrm{~mm}$ in the mild injury group, $25.0 \mathrm{~mm}$ in the moderate injury group, $50.0 \mathrm{~mm}$ in the severe injury group) and released the falling rod to induce the spinal cord injury.

\section{Measurement of intramedullary pressure}

The intramedullary pressure was measured by a Millar Mikro-Tip pressure catheter immediately after laminectomy in the control group or at different time points after the injury in the experimental groups and recorded by a PowerLab system (AD Instruments Pty Ltd, Bella Vista, NSW, Australia). The spine of each rat was fixed with a spinous process clip and then with a stereotaxic instrument (ZH-Blue Star B, Huaibei Zhenghua Biological Equipment Company, Huaibei, China); the spinal dura was penetrated by a $22 \mathrm{G}$ venipuncture cannula $30^{\circ}$ parallel to the horizontal plane of the spine. The puncture point was $2.5 \mathrm{~mm}$ caudal to the impact site on the inclined left or right of the surface to avoid the posterior median artery (right or left of T11 in the control group). As soon as the tip of tube was just over the spinal dura, the venipuncture cannula probe was extracted and the pressure catheter was inserted through the tube and into the spinal cord until $3 \mathrm{~mm}$ extruded out of the tube (Figure 2). The pressure was recorded for $1 \mathrm{~min}$. After the measurements, the rats were killed by cervical dislocation under anesthesia.

\section{Statistical analysis}

The statistical analysis was performed by the SPSS software (Version 17.0), SPSS Company, Chicago, IL, USA. The data are expressed as mean \pm s.d. The data of the experimental group were analyzed by factorial analysis to detect the effects of injury degree and time on intramedullary pressure. Then, the onefactor analysis of variance was used to analyze the differences in intramedullary pressure at different times for the same injury degree. Rank sum test was used to compare the data of the experimental and control groups. The correlation between intramedullary pressure and injury degree over time was analyzed by multiple linear correlation. Values of $P<0.05$ were considered statistically significant.

We certify that all applicable institutional and governmental regulations concerning the ethical use of animals were followed during the course of this research.

\section{RESULTS}

The average intramedullary pressure of rats in the control group was $6.88 \pm 1.67 \mathrm{~mm} \mathrm{Hg}$. In the experimental group, after the impact, the rats' tails convulsively swung immediately, the bilateral hind limbs appeared flaccidly paralyzed after a retracted flutter and the data (impact velocity, cord compression distance, cord compression time and cord compression rate) measured and displayed by computers met the requirement, so the models were all successful. The intramedullary pressures of the rats in the experimental group by different degrees of injury at different times are shown in Table 1. The line chart drawn by the GraphPad Prism software (Version 5, GraphPad Software Incorporated Company, La Jolla, CA, USA) is shown in Figure 1. The results of rank sum test showed that the

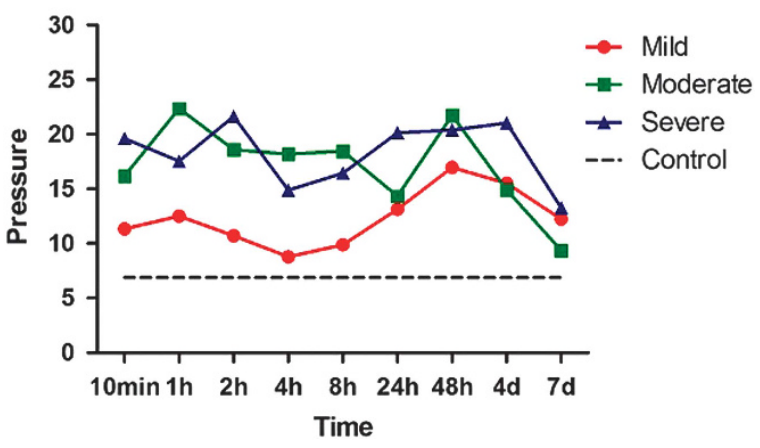

Figure 1 Line chart of intramedullary pressure over time in three experimental groups. The curves of intramedullary pressure over time in the mild and moderate injury groups are bimodal, as the intramedullary pressure peaked at 1 and $48 \mathrm{~h}$ after the injury, whereas the curve of intramedullary pressure over time in the severe group is relatively flat. They are all above the control line.

intramedullary pressure of the rats in any injury group was significantly higher than that in the control group $(P=0.000)$. The factorial analysis showed that both injury degree and time influenced intramedullary pressure $(P=0.000$ and 0.004 , respectively). The variance analysis of data in the mild/moderate injury group showed a statistically significant difference in intramedullary pressure among the different time points $(P=0.007 / 0.017)$. Figure 1 shows that the curves of intramedullary pressure over time in the mild and moderate injury group are both bimodal, peaking at 1 and $48 \mathrm{~h}$ after the injury. However, the curve of intramedullary pressure in the severe injury group was relatively flat over time in Figure 1. The variance analysis showed no statistically significant differences among the different time points in the severe injury group $(P=0.374)$. The correlation between intramedullary pressure and injury degree over time was analyzed by the multiple linear correlation. The partial correlation coefficient was $0.438(P=0.000)$, suggesting that intramedullary pressure was positively correlated with injury degree, that is, the more serious the injury degree, the higher the intramedullary pressure.

\section{DISCUSSION}

\section{Intramedullary pressure measurements}

There have been only two reports to date with numerical intramedullary pressure results. Saadoun ${ }^{5}$ used a Millar pressure catheter to determine that the normal intramedullary pressure of mice is $8 \pm 3 \mathrm{~mm} \mathrm{Hg}$. Iida $^{6}$ determined that the normal intramedullary pressure of hybrid dogs is $30 \pm 12 \mathrm{~mm} \mathrm{Hg}$. This large difference may be because of the different species. This experiment showed that the normal intramedullary pressure of rats is $6.88 \pm 1.67 \mathrm{~mm} \mathrm{Hg}$, which is similar to the results in the previous studies of mice.

Compared with the balloon method applied by Iida, ${ }^{6}$ the Millar pressure catheter used both here and by Saadoun ${ }^{5}$ was a solid-state 


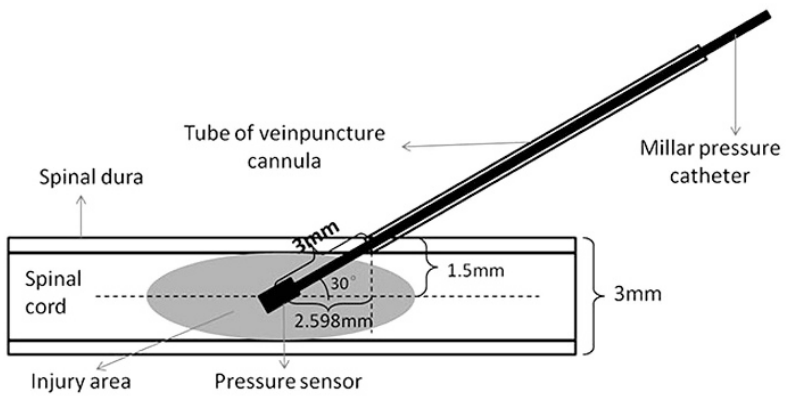

Figure 2 The ideograph of measuring the intramedullary pressure. The spinal dura was penetrated by a $22 \mathrm{G}$ venipuncture cannula $30^{\circ}$ parallel to the horizontal plane of the spine. The puncture point was $2.5 \mathrm{~mm}$ behind the impact site on the inclined left or right of the surface to avoid the posterior median artery (right or left of T11 in the control group). As soon as the tip of tube was just over the spinal dura, the venipuncture cannula probe was extracted and the pressure catheter was inserted through the tube and into the spinal cord until $3 \mathrm{~mm}$ extruded out of the tube.

pressure sensor with higher accuracy $(0.1 \mathrm{~mm} \mathrm{Hg})$ and a diameter of only $0.46 \mathrm{~mm}$, meaning that it caused little tissue injury. We inserted the catheter at an angle of $30^{\circ}$, which extended the path and minimized error. The puncture point was $2.5 \mathrm{~mm}$ caudal to the impact site on the inclined left or right of the surface to avoid the posterior median artery, and the puncture depth was $3 \mathrm{~mm}$ (the diameter of the thoracic segmental spinal cord of rats was approximately $3 \mathrm{~mm}$ in the literature ${ }^{7}$ ), to ensure that the pressure sensor on the tip of catheter was located in the central area of the spinal cord injury (Figure 2). The measurement in the control group was similar and the puncture point was on the inclined left or right of the surface of T11. Because the tip of the Millar Mikro-Tip pressure catheter was round and blunt, the venipuncture cannula was used to penetrate the spinal dura and play a guiding role. In the preliminary experiments, we found that the pressure remained stable in the initial $1 \mathrm{~min}$ in both the control and experimental groups after catheter insertion, and thus we recorded the average continuous pressure in the initial $1 \mathrm{~min}$ post insertion.

\section{Intramedullary pressure changes over time after traumatic spinal cord injury}

Only one study to date reported on the intramedullary pressure after spinal cord contusion injury. Using a Millar pressure catheter, Saadoun ${ }^{5}$ found that the intramedullary pressure was as high as $27 \pm 3 \mathrm{~mm} \mathrm{Hg}$ at $48 \mathrm{~h}$ after the contusion injury of spinal cord, which was higher than our results $(16.97 \pm 4.33,21.73 \pm 7.73$ and $20.40 \pm 6.29 \mathrm{~mm} \mathrm{Hg}$ for mild, moderate and severe, respectively).

The main factors causing the intramedullary pressure to increase after traumatic spinal cord injury were intramedullary hemorrhage and edema.

Weirich ${ }^{8}$ found scattered hemorrhages in the gray matter of the spinal cord at $2 \mathrm{~h}$ after injury that quickly spread and peaked at $12 \mathrm{~h}$. Wagner ${ }^{9}$ found hemorrhaging in the gray matter at 15 min after injury that spread across the entire gray matter within $4 \mathrm{~h}$. Osterholm ${ }^{10}$ found dotted hemorrhaging in the gray matter within $15 \mathrm{~min}$ after injury that expanded quickly and accounted for $70 \%$ of the crosssectional area of the spinal cord at $24 \mathrm{~h}$. Leonard ${ }^{11}$ found that hemorrhaging in the injured area peaked $5 \mathrm{~h}$ after the spinal cord injury and then decreased. In the above studies, intramedullary hemorrhaging peaked within hours after spinal cord injury, and thus we speculated that intramedullary hemorrhaging was the main cause of the intramedullary pressure increases soon after spinal cord injury.
As the hemorrhaging was controlled and absorbed, the pressure decreased slightly, which induced a pressure peak at $1 \mathrm{~h}$ in the mild and moderate groups of this study. There was no pressure peak in the severe injury group in our experiment, because the hemorrhaging was severe and not easy to control and absorb.

Dohrmann ${ }^{12}$ found that the edema of nerve fibers appeared within 15-20 min and increased gradually within 1-6 h after injury. Yashon ${ }^{13}$ observed that edema of the spinal cord arose within 5 min after injury that peaked at 5 days and then gradually declined. Leonard ${ }^{11}$ observed that edema in the spinal cord increased quickly within $5 \mathrm{~h}$ after injury, and then gradually increased and peaked at 1 week. Torre ${ }^{14}$ pointed out that 2-3 days after injury was the most serious period of edema. $\mathrm{Hu}^{15}$ found that the water content of the spinal cord was the most at 2 days after injury. Weirich ${ }^{8}$ found that edema in rats was most serious within 48 and $72 \mathrm{~h}$ after mild spinal cord injury, whereas the edema was still developing at 72-96 h after the severe injury, which explained the phenomenon in this experiment that, after severe injury, intramedullary pressure was always high and did not appear to peak by 7 days. In the above literature, the edema was developed gradually and did not decrease until 2-7 days after the injury, and thus we speculated that the effect of edema on the increases in intramedullary pressure lasted for the acute phase after the injury, which explains the second pressure peak seen in the mild and moderate groups.

Correlation between the intramedullary pressure and injury degree This study proved that intramedullary pressure and injury degree were positively correlated. No study to date has examined the correlation between intramedullary pressure and injury degree after spinal cord contusion injury. Researchers have proven that intramedullary pressure was correlated with the degree of stretch after the spinal cord stretch injury. Iida ${ }^{6}$ found that the intramedullary pressure of hybrid dogs increased rapidly after the spinal cord stretch injury and that intramedullary pressure and tensile strength were linearly related. In an autopsy study, Chavanne ${ }^{16}$ found that intramedullary pressure rose significantly when the posterior protrusion deformity of the cervical spine was $>21^{\circ}$.

It has been proven that the amount of intramedullary hemorrhage and the extent of edema are correlated with injury degree. Ducker ${ }^{17}$ found that intramedullary hemorrhage amount and injury degree were positively correlated in monkeys. Torre ${ }^{14}$ pointed out that edema degree was related to injury degree and that the greater the injury, the more serious the edema. If intramedullary pressure was associated with intramedullary hemorrhage and edema extent, the above documents could also be indirect proof that intramedullary pressure is associated with injury degree.

\section{Deficiencies}

This experiment also had some disadvantages. First, in this study, we performed a laminotomy to expose the spinal cord, so the pressure measurement results may be affected by the operation and anesthesia. Second, the size of each group was small, and thus the sampling error was large. Third, because of the invasive measurements and the animals' activity, we could not continue monitoring the pressure. Thus, rats can only be measured once, and thus the individual difference between animals was one factor that influenced the accuracy of the experimental results. Finally, there is a lack of histology. The intramedullary pressure readings could not be directly correlated with injury pathology, because the catheter disrupts local histology. 


\section{CONCLUSION}

In rats, the intramedullary pressure increased after traumatic spinal cord injury. If the injury was not serious, the intramedullary pressure fluctuated with time and peaked at 1 and $48 \mathrm{~h}$ after injury. If the injury was serious, the intramedullary pressure remained at a high level. The more serious the traumatic spinal cord injury, the higher the intramedullary pressure.

\section{DATA ARCHIVING}

There were no data to deposit.

\section{CONFLICT OF INTEREST}

The authors declare no conflict of interest.

\section{ACKNOWLEDGEMENTS}

This work was supported by the National Natural Science Foundation of China (81272164) and the Special Fund for Basic Scientific Research of Central Public Research Institutes (2014CZ-20).

1 Jägersberg M, Schaller C, Boström J, Schatlo B, Kotowski M, Thees C. Simultaneous bedside assessment of global cerebral blood flow and effective cerebral perfusion pressure in patients with intracranial hypertension. Neurocrit Care 2010; 12: 225-233.

2 Salinas NM. Ocular hypertension impairs optic nerve axonal transport leading to progressive retinal ganglion cell degeneration. Exp Eye Res 2010; 90: 168-183.
3 Ouyang H, Galle B, Li J, Nauman E, Shi R. Biomechanics of spinal cord injury: a multimodal investigation using ex vivo guinea pig spinal cord white matter. J Neurotrauma 2008; 25: 19-29.

4 Young W. Spinal cord contusion models. Prog Brain Res 2002; 137: 231-255.

5 Saadoun S, Bell B, Verkman A, Papadopoulos M. Greatly improved neurological outcome after spinal cord compression injury in AQP4-deficient mice. Brain 2008; 131: 1087-1098.

6 Iida H, Tachibana S. Spinal cord intramedullary pressure: direct cord traction test. Neurol Med Chir 1995; 35: 75-77.

7 Waibl H. Zur Topographie der Medulla spinalis der Albinoratte (Rattus norvegicus). Adv Anat Embryol Cell Biol 1973; 47: 5-42.

8 Weirich SD, Cotler HB, Narayana PA, Hazle JD, Jackson EF, Coupe KJ et al. Histopathologic correlation of magnetic resonance imaging signal patterns in a spinal cord injury model. Spine 1990; 15: 630-638.

9 Wagner FC Jr, Dohrmann GJ, Bucy PC. Histopathology of transitory traumatic paraplegia in the monkey. J Neurosurg 1971; 35: 272-276.

10 Osterholm JL. Spinal pathways mediating traumatic hemorrhagic necrosis. Trans Am Neurol Assoc 1972; 97: 187

11 Leonard AV, Thornton E, Vink R. The relative contribution of edema and hemorrhage to raised intrathecal pressure following traumatic spinal cord injury. J Neurotrauma 2015; 32: 397-402.

12 Dohrmann GJ, Wagner FC Jr, Bucy PC. The microvasculature in transitory traumatic paraplegia: an electron microscopic study in the monkey. J Neurosurg 1971; 35: 263-271.

13 Yashon D, Bingham WG Jr, Faddoul EM, Hunt WE. Edema of the spinal cord following experimental impact trauma. J Neurosurg 1973; 38: 693-697.

14 Torre JCDL. Spinal cord injury: review of basic and applied research. Spine 1981; 6: 315-335.

15 Hu A-M, Li J-J, Sun W, Yang D-G, Yang M-L, Du L-J et al. Myelotomy reduces spinal cord edema and inhibits aquaporin-4 and aquaporin-9 expression in rats with spinal cord injury. Spinal Cord 2015; 53: 98-102.

16 Chavanne A, Pettigrew DB, Holtz JR, Dollin N, Kuntz Cl. Spinal cord intramedullary pressure in cervical kyphotic deformity: a cadaveric study. Spine 1976; 36: 1619-1626.

17 Ducker T, Kindt G, Kempe L. Pathological findings in acute experimental spinal cord trauma. J Neurosurg 1971; 35: 700-708. 